[0212-7199 (2008) 25: 5; pp 222-225] ANALES DE MEDICINA INTERNA Copyright (C) 2008 ARAN EDICIONES, S.L.

AN. MED. INTERNA (Madrid) Vol. 25, N. $^{\circ} 5$, pp. $222-225,2008$

\section{Uso de la espirometría versus ecocardiografía en pacientes hospitalizados por EPOC o insuficiencia cardiaca}

\author{
M. J. TORRUBIA FERNÁNDEZ, A. COMÍN ORCE, M. MORENO VERNIS, \\ S. J. CARRIZO SIERRA, J. M. MARÍN TRIGO
}

Servicios de Medicina Interna, Documentación y Neumología. Hospital Universitario Miguel Servet. Zaragoza
SPIROMETRY AND ECHOCARDIOGRAPHY USE IN HOSPITALISED PATIENTS DUE TO COPD OR HEART FAILURE

\section{RESUMEN}

Objetivo: El uso de la prueba complementaria confirmadora del diagnóstico de EPOC (espirometría) a nivel hospitalario es desconocido. Se pretende determinar el uso de la espirometría (ESPIR) y comparativamente de la ecocardiografía (ECO) en la insuficiencia cardiaca (IC) en pacientes ingresados por exacerbación de estas entidades.

Métodos: Se incluyeron pacientes que a lo largo de un año fueron dados de alta con los diagnósticos primarios de "exacerbación de EPOC" y de "insuficiencia cardiaca" (códigos ICD-9-CM: 491.21 y 428, respectivamente). A partir del listado, se revisaron todas las historias clínicas y se obtuvieron los datos originales de las ESPIR y de las ECO de los gabinetes donde se realizaron las pruebas.

Resultados: De 408 pacientes ingresados por EPOC, se realizó ESPIR en $110(27 \%)$ y de los 328 pacientes con IC se obtuvo ECO en $250(76 \%)(\mathrm{p}<0,001)$. Salvo una menor edad de los pacientes con EPOC $(71,8 \pm 9,8 v s .74,9 \pm 10,6, \mathrm{p}<0,001)$, no hubo diferencias entre ambos grupos. El subgrupo en que se realizó ESPIR, mostró una edad mas baja $(69,5 \pm 9,1$ vs. $72,5 \pm 9,9, \mathrm{p}=0,005)$ y un \% de ingresos en Neumología respecto a Medicina Interna mas alto $(30 v s .13 \%, \mathrm{p}<0,002)$, respecto al subgrupo sin ESPIR.

Conclusiones: Se evidencia un escaso uso de la espirometría en pacientes ingresados por exacerbación de EPOC. La baja prevalencia de prescripción de esta prueba confirmatoria comparativamente con la alta prescripción de la ecocardiografía para pacientes ingresados por IC, parece indicar que este comportamiento es enfermedad-específico.

PALABRAS CLAVE: Espirometría. Ecocardiografía. Enfermedad pulmonar obstructiva crónica. Hospitalización. Uso de recursos sanitarios.

\section{ABSTRACT}

Background and objective: There is no information on the use of the spirometry as a confirmatory tool for the diagnosis of COPD at the hospital setting. To determine the pattern of use of the spirometry (SPIR) in comparison with the use of the echocardiography $(E C H O)$ in heart failure $(H F)$.

Methods: We included those patients that during one year they were discharge with the primary diagnosis of "COPD exacerbation" or "Heart Failure" (codes: ICM-9-CM: 491.21 y 428). From the generated list, history charts were reviewed and original SPIR and ECHO were pooled out from the pulmonary function test and non-invasive cardiac unit departments.

Results: Among 408 patients with COPD, SPIR was done in 110 (27\%) and among 328 patients with $\mathrm{HF}, \mathrm{ECHO}$ was prescribed in 250 $(76 \%)(p<0,001)$. COPD group was younger $(71.8 \pm 9.8 v s .74 .9 \pm$ 10.6, $p<0,001)$, but no other differences were found with HF group. Among those patients who had SPIR, age was lower $(69.5 \pm 9.1$ vs. 72.5 $\pm 9.9, p=0,005)$ and there was a high \% of in-patients carried by pulmonology versus internal medicine (30vs. $13 \%, p<0,002)$ comparing with the group of patients who had no SPIR.

Conclusions: We found a small percentage of COPD patients who had spirometry during hospitalization due to COPD exacerbation. This peculiar pattern of the limited use of the confirmatory diagnostic test appear disease-specific since we also found a large percentage of patient with heart failure who had echocardiography during their hospitalization.

KEY WORDS: Spirometry. Echocardiography. Chronic obstructive pulmonary disease. Heart failure. Health care resources utilization.

Torrubia Fernández MJ, Comín Orce A, Moreno Vernis M, Carrizo Sierra SJ, Marín Trigo JM. Uso de la espirometría versus ecocardiografía en pacientes hospitalizados por EPOC o insuficiencia cardiaca. An Med Interna (Madrid) 2008; 25: 222-225.

\section{INTRODUCCIÓN}

La enfermedad pulmonar obstructiva crónica (EPOC) afecta en España a un 9\% de la población y la insuficiencia cardiaca (IC) a otro $6 \%(1,2)$. Se sabe que en el caso de la EPOC, la enfermedad está infradiagnosticada de forma que sólo el $22 \%$ de los pacientes tiene confirmado su padecimien- to mediante la realización de una espirometría. Esta prueba no solo confirma el diagnóstico, sino que establece la severidad del proceso y orienta la estrategia terapéutica $(3,4)$. La no disponibilidad de espirometría en un paciente con sospecha de EPOC impide confirmar el diagnóstico, puede retrasar un tratamiento específico y determina finalmente un peor pronóstico. La EPOC y la IC consumen enormes recursos sanitarios y

Trabajo aceptado: 18 de enero de 2008 
específicamente son las dos causas mas frecuentes de ingresos médicos en hospitales generales (5).

La hospitalización por exacerbación de la EPOC o de la IC son un punto de inflexión en la historia natural de ambas entidades ya que empeora su pronóstico $(6,7)$. Por el contrario el ingreso hospitalario puede permitir una evaluación exhaustiva de los pacientes y confirmar el diagnóstico correcto de las entidades. La realización de espirometría y ecocardiografía es recomendada por guías de buena practica clínica porque pueden determinar un mejor manejo ulterior de la EPOC y la IC respectivamente $(3,8)$. En el caso de la EPOC, este aspecto es especialmente relevante porque la mortalidad de la enfermedad sigue aumentando (9). Entre las causas que explicarían este incremento se aduce precisamente el escaso uso de la espirometría y por tanto el desconocimiento de la severidad del proceso con el ajuste terapéutico consiguiente.

Nuestra impresión en la práctica clínica, es que el uso de la espirometría es limitado incluso en pacientes hospitalizados por exacerbación de EPOC comparativamente con otras pruebas complementarias confirmatorias de diagnóstico como sería el caso de la ecocardiografía para la IC. Para comprobar esta hipótesis realizamos un estudio retrospectivo sobre el uso de la espirometría y de la ecocardiografía en pacientes hospitalizados por exacerbaciones de EPOC y de IC respectivamente en un hospital terciario.

\section{MÉTODOS}

\section{PACIENTES}

El estudio fue desarrollado en el Hospital Universitario Miguel Servet de Zaragoza. El centro hospitalario, de tercer nivel, sirve como centro de referencia para una población de unas 650,000 personas. A partir de la base de altas codificadas del Hospital, se identificaron los pacientes que tuvieron un diagnóstico de EPOC y de insuficiencia cardiaca como causas primarias de ingreso entre 1 de enero de 2005 y 31 de Diciembre de 2005. Con base en la Clasificación Internacional de Enfermedades, en su $9^{\text {a }}$ versión modificada clínica (ICD-9CM), se utilizaron los códigos 491.21 para EPOC con exacerbación y los códigos 428 a 428.9 para insuficiencia cardiaca. Una vez establecidos los listados de potenciales candidatos a estudio, fueron excluidos aquellos pacientes que fueron también estaban etiquetados en las altas hospitalarios con diagnósticos secundarios de neumonía, asma, fibrosis pulmonar, cáncer de pulmón u otra neumopatía asociada.

\section{PROCEDIMIENTOS}

El listado definitivo de pacientes con diagnóstico primario de alta de EPOC o IC fue utilizado para evaluar en el Laboratorio de Función Pulmonar y en la Unidad de Ecocardiografía la realización de pruebas funcionales y de ecocardiografía respectivamente a los pacientes durante el periodo de inclusión. En el caso de la espirometría, la prueba se realiza según recomendaciones predeterminadas (10) y los valores obtenidos se comparan con las tablas de normalidad para la población mediterránea (11). En el caso de la ecocardiografía, la exploración durante este periodo ha sido realizada por 2 cardiólogos expertos y según procedimientos estandarizados (12).
La historia clínica de cada paciente fue revisada y a partir de ella se registraron las pruebas complementarias realizadas en cada ingreso hospitalario y los procedimientos terapéuticos aplicados. En una base de datos específicamente desarrollada al efecto, se incluyó la edad, sexo, fechas de ingreso y alta, comorbilidad, necesidad de tratamiento intensivo y morbimortalidad. Dado que se trata de un estudio relacionado con la calidad asistencial, el Comité de Ética e Investigación Clínica del centro no requirió de la necesidad de consentimiento informado por parte de los pacientes.

\section{ANÁLISIS ESTADÍSTICO}

El resumen de la descripción analítica de las poblaciones estudiadas se presenta utilizando frecuencias y proporciones para las variables categóricas y mediante medias y desviaciones típicas para las variables continuas. Las diferencias entre grupos de las diferentes variables cuantitativas o categóricas estudiadas, se estudió mediante el método de t de Student para datos no pareados y de $\chi^{2}$ respectivamente. Un valor de $\mathrm{p}<$ 0,05 fue considerado como estadísticamente significativo. Todos los análisis fueron realizados mediante el paquete estadístico SPSS versión 14.

\section{RESULTADOS}

A lo largo del periodo de inclusión del presente estudio se registraron 673 altas con diagnóstico primario de exacerbación de EPOC correspondientes a 408 pacientes y 511 altas con diagnóstico primario de IC correspondientes a 328 pacientes (Tabla I). El grupo con diagnóstico de IC mostró mayor edad $(74,9 \pm 10,6$ vs. 71,8 \pm 9,8, p < 0,001), incluyó un mayor porcentaje de mujeres (49vs. $11 \%, \mathrm{p}<0,0001)$ y fue ingresado con mayor probabilidad en el Servicio de Medicina Interna -MI- (37vs. $21 \%, \mathrm{p}<0,001)$ respecto al grupo con diagnóstico de EPOC. Ambos grupos no se diferenciaron en el grado de comorbilidad medido por el índice de Charlson, la duración de la estancia hospitalaria o la propia mortalidad intra-hospitalaria. La historia clínica revisada de todos los sujetos mostró que a solo un $21 \%$ de EPOC se les había realizado una espirometría antes del presente ingreso frente a un

\section{TABLA I}

\section{CARACTERÍSTICAS DE LOS PACIENTES}

\begin{tabular}{lccc}
\hline & EPOC & IC & $P$ \\
\hline$N^{0}$ de pacientes & 408 & 328 & \\
$\mathrm{~N}^{0}$ de hospitalizaciones & 673 & 511 & \\
Edad, años & $71,8 \pm 9,8$ & $74,9 \pm 10,6$ & $<0,001$ \\
Sexo, hombres/mujeres & $363 / 45$ & $166 / 162$ & $<0,001$ \\
Comorbilidad, Índice de Charlson & $2,9 \pm 1,3$ & $3,1 \pm 1,5$ & 0,06 \\
Estancia media, días & $10,9 \pm 8,1$ & $11,1 \pm 8,3$ & 0,76 \\
Servicio de ingreso: & & & \\
$\quad$ Neumología o cardiología vs. MI & $529 / 140$ & $301 / 189$ & $<0,001$ \\
Mortalidad intrahospitalaria, n (\%) & $16(3,9)$ & $22(6,7)$ & 0,12 \\
ESPIR o ECO previa en la historia, n (\%) & $86(21)$ & $256(78)$ & $<0,001$ \\
\hline
\end{tabular}

EPOC: enfermedad pulmonar obstructiva crónica; IC: insuficiencia cardiaca congestiva; MI: Medicina Interna; ESPIR: espirometría; ECO: ecocardiografía. 
$78 \%$ de pacientes con IC a los que se les había realizado una ecocardiografía $(\mathrm{p}<0,001)$.

A partir de las historias clínicas y de su correspondiente comprobación en los archivos de los gabinetes de pruebas funcionales y de ecocardiografía, resultó que a 110 pacientes (27\%) de grupo EPOC y a 250 pacientes (76\%) del grupo IC, se les practicó espirometría o ecocardiografía respectivamente durante su hospitalización ( $\mathrm{p}<0,001)$ (Fig. 1). Agrupando las espirometría y las ecocardiografias realizadas previamente a la hospitalización y disponibles en la historia clínica, resultó que finalmente, sólo un 39\% de pacientes con EPOC disponían de espirometría frente a un $88 \%$ de pacientes con IC a los que se les realizó al menos una ecocardiografía. En la tabla II aparecen las características de ambos grupos de pacientes según se les hubiese realizado o no la prueba complementaria. En los pacientes con IC, no hubo diferencias entre ambos subgrupos. En el grupo de pacientes con EPOC, el subgrupo al que se le indicó espirometría durante su hospitalización, tenia una edad inferior ( $69,5 \pm 9,1 \mathrm{vs} .72,5 \pm 9,9, \mathrm{p}=0,005)$ y fueron ingresados con menor probabilidad en medicina interna respecto a Neumología (13vs. 30\%, p <0,002), respecto a los pacientes que no dispusieron de esta prueba. No encontramos otras diferencias clínicas, analíticas o de curso evolutivo entre ambos subgrupos.

\section{DISCUSIÓN}

El presente estudio es el primero en evaluar, al menos en nuestro país, el uso de la espirometría y de la ecocardiografía durante la hospitalización en pacientes con diagnóstico primario de alta de EPOC e IC respectivamente. Dos son los resultados destacados de nuestro trabajo. Por un lado la demostración del escaso uso de la espirometría como prueba complementaria para confirmar el diagnóstico y severidad de los pacientes con EPOC que ingresan en el hospital por una agudización de su enfermedad. El otro aspecto relevante es que este déficit de indicación de la espirometría como prueba complementaria a nivel hospitalario parece estar relacionado con la propia enfermedad: la EPOC. En otra entidad clínica como la IC, con muchas similitudes en cuanto a cronicidad y uso de recursos sanitarios, el uso de la ecocardiografía como prueba complementaria es mucho mayor. Globalmente sólo un $39 \%$ de pacientes ingresados por EPOC dispusieron de
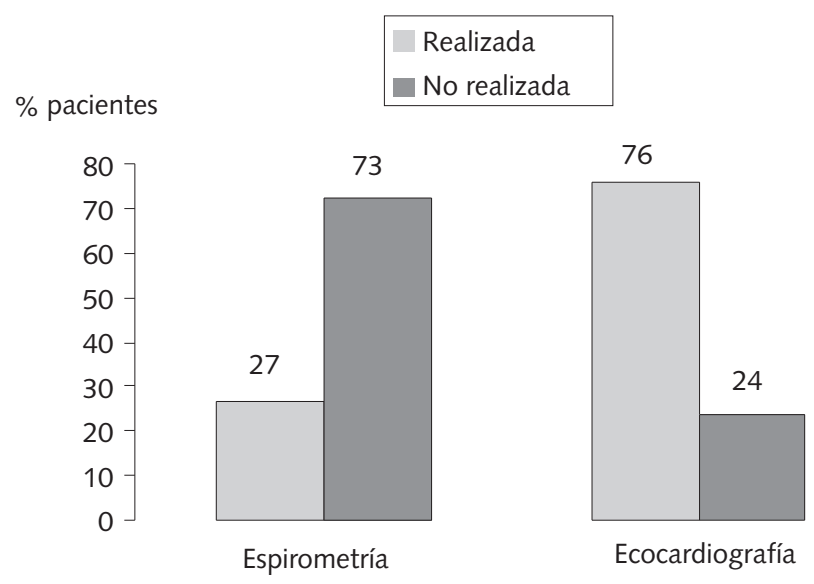

Fig. 1. Porcentaje de pacientes con diagnóstico final de EPOC a los que les fue realizada (en color claro) o no realizada (en color oscuro) espirometría, en comparación con el porcentaje de pacientes con diagnóstico final de Insuficiencia cardiaca, a los que les fue realizada (en color claro) o no realizada (color oscuro) ecocardiografía durante el ingreso hospitalario.

espirometría frente a un $88 \%$ de pacientes con IC que disponían de ecocardiografía. Este patrón de uso de la espirometría en pacientes ingresados por EPOC no parece peculiar de nuestro centro. Nuestros hallazgos son muy similares a los de Damarla y cols. en EE.UU. (13). Estos autores estudiaron de forma retrospectiva una población de pacientes con EPOC y con IC ingresados a lo largo de 6 meses por agudización de su enfermedad. Al analizar las pruebas complementarias realizadas, encontraron que a los pacientes con EPOC solo se les practicó espirometría en un $31 \%$ de casos mientras que se realizó ecocardiografía a un $78 \%$ de casos con IC.

El diseño de nuestro estudio no permite conocer las causas aparentes del escaso uso de la espirometría en medio hospitalario en pacientes con EPOC, pero permite no obstante descartar algunos potenciales responsables. Por ejemplo, no hubo diferencias en las dificultades logísticas para el acceso a las pruebas diagnósticas en los gabinetes de espirometría y eco-

TABLA II

CARACTERÍSTICAS DE LOS PACIENTES CON EPOC Y CON ICC SEGÚN SE HAYA REALIZADO O NO, ESPIROMETRÍA O ECOCARDIOGRAFÍA Y SERVICIO QUE SOLICITÓ LA EXPLORACIÓN COMPLEMENTARIA

\begin{tabular}{lcccccc}
\hline \multicolumn{3}{c}{ EPOC } & \multicolumn{3}{c}{ ICC } \\
\hline & ESPIR sí & ESPIR no & $p$ & ECO sí & ECO no & $p$ \\
\hline$N^{\circ}$ pacientes (\%) & $110(27)$ & $298(73)$ & $<0,001$ & $250(76)$ & $78(24)$ & $<0,001$ \\
Edad, años & $69,5 \pm 9,1$ & $72,5 \pm 9,9$ & 0,005 & $74,2 \pm 11,8$ & $75,2 \pm 12,6$ & 0,27 \\
Hombres/mujeres, $n^{\circ}$ & $104 / 6$ & $259 / 39$ & 0,04 & $131 / 119$ & $35 / 43$ & 0,30 \\
Índice de Charlson & $2,8 \pm 1,5$ & $2,9 \pm 1,2$ & 0.53 & $3,1 \pm 1,6$ & $3,1 \pm 1,5$ & 0,99 \\
Estancia media, días & $10,1 \pm 8,8$ & $11,7 \pm 7,3$ & 0,06 & $10,5 \pm 9,3$ & $11,5 \pm 9,3$ & 0,40 \\
Servicio a cargo del paciente: & & & & & & \\
$\quad$ Neumología o Cardiología vs. MI 97/10 & $223 / 68$ & $<0,002$ & $155 / 90$ & $46 / 30$ & 0,68 \\
\hline
\end{tabular}

EPOC: enfermedad pulmonar obstructiva crónica; IC: insuficiencia cardiaca congestiva; MI: Medicina Interna; ESPIR: espirometría; ECO: ecocardiografía. 
cardiografía. Ambos grupos de pacientes (IC y EPOC) presentaban similares grados de comorbilidad y estancia media. Incluso los pacientes con EPOC eran ligeramente menos mayores. Las diferencias encontradas en el porcentaje de indicación entre neumólogos e internistas para la espirometría (7 vs. $18 \%$ ) respecto al comportamiento de cardiólogos e internistas con la ecocardiografía (52 vs. 48\%), tampoco justifica el escaso uso de la primera. La realidad del escaso uso de la espirometría en medio hospitalario, también se evidencia a nivel de atención primaria. Recientemente, Naberan y cols. comunican que sólo un 59\% de centros de salud disponen de espirómetros, pero aun en estos, el uso de la espirometría es escasa por diversa razones, especialmente el déficit de formación en el uso de la prueba (14). En Gran Bretaña, a nivel de atención primaria, los porcentajes de uso de la espirometría en el manejo de pacientes con EPOC muestran una media entre centros del $37 \%$ (15). En Norteamérica también se ha detectado esta infrautilización de la espirometría. En una revisión de las pruebas complementarias médicas realizadas a más de un millón y medio de personas adheridas a un seguro médico, se encontró que solo en un $32 \%$ de los 5.039 pacientes diagnosticados de EPOC en los 2 años anteriores, se les practicó espirometría para confirmar el diagnóstico (16).

La no realización de espirometría es lesiva para el paciente en dos aspectos fundamentales: a) la demostración de la presencia de un déficit ventilatorio obstructivo, determina el diagnóstico de la EPOC y excluye una enfermedad pulmonar restrictiva que tiene un enfoque terapéutico radicalmente diferente; y b) la categorización de la severidad de la obstrucción determina el pronóstico de la enfermedad y su tratamiento $(3,4)$. Miravitlles y cols. han puesto de manifiesto la gravedad de la no disponibilidad de espirometría en pacientes con EPOC (17). En un estudio amplio de pacientes con EPOC atendidos en centros de AP, sólo un 47\% disponía de espirometría. En el seguimiento prospectivo de estos sujetos, pudo comprobarse como aquellos pacientes que aún con severidad similar de la enfermedad no disponían de espirometría, eran mas proclives a exacerbaciones y visitas no programadas a centros de AP y urgencias hospitalarias respecto a los que disponían de función pulmonar obtenida por espirometría.

Existe pues una aparente contradicción entre las recomendaciones de las guías nacionales e internacionales del manejo de la EPOC que recomiendan la realización de espirometría como elemento imprescindible en el manejo de enfermos con EPOC, y la realidad del escaso uso de esta prueba en la práctica clínica. Nuestro trabajo sugiere que el escaso uso de la espirometría a nivel hospitalario no se debe a limitaciones técnicas, formativas o de disponibilidad de la prueba sino apunta mas bien a la ausencia de recomendaciones mas claras sobre cuando y con que frecuencia se debe practicar la espirometría y como sus resultados deben implementar el tratamiento de los pacientes con exacerbación severa de EPOC.

\section{Bibliografía}

1. Sobradillo V, Miravitlles M, Jiménez-Ruiz CA, Gabriel R, Viejo JL, Masa JF et al. Epidemiological study of chronic obstructive pulmonary disease in Spain (IBERPOC): prevalence of chronic respiratory symptoms and airflow limitation. Arch Bronconeumol 1999; 35: 159-66.

2. Muñiz Garcia J, Crespo Leiro MG, Castro Beiras A. Insuficiencia cardiaca en España- Epidemiología e importancia del grado de adecuación a las guis de practica clínica. Rev Esp Cardiol 2006; 6: 2-8.

3. Celli BR, MacNee W. Standards for the diagnosis and treatment of patients with COPD: a summary of the ATS/ERS position paper. Eur Respir J 2004; 23: 932-46.

4. GOLD executive committee. Global Initiative for Chronic Obstructive Pulmonary Disease. 2006. Consultado 27.07.07; acceso en: www.GOLD.com .

5. Instituto Nacional de Estadística.. Encuesta de Morbilidad Hospitalaria. Año 2002. Madrid: Instituto Nacional de Estadística; 2005.

6. Sánchez AM, los investigadores del Registro BADAPIC. Características clínicas, tratamiento y morbimortalidad a corto plazo de pacientes con insuficiencia cardiaca controlados en consultas específicas de insuficiencia cardiaca. Resultados del Registro BADAPIC. Rev Esp Cardiol 2004; 57: 1159-69.

7. Donaldson GC, Seemungal TA, Bhowmik A. Relationship between exacerbation frequency and lung function decline in chronic obstructive pulmonary disease. Thorax 2002; 57: 847-852.

8. Moreo A, de Chiara B, Cataldo G, Pícalo G. Lobiati E. Parolini M, et al. Valor pronóstico de las medidas seriadas de la función ventricular izquierda y del rendimiento en el ejercicio en la insuficiencia cardiaca crónica. Rev Esp Cardiol. 2006; 59: 905-10.

9. Chapman KR, Mannino DM, Soriano JB. Epidemiology and costs of chronic obstructive pulmonary disease. Eur Respir J 2006 ; 27: 188 207.

10. Sanchis J, Casan P, Castillo J, González N, Palenciano L, Roca J. Normativa para la espirometría forzada. Recomendaciones SEPAR 1. Arch Bronconeumol 1989; 25: 132-142.

11. Roca J, Sanchos J, Agusti-Vidal A. Spirometric referente values for a meditarranean population. Bull Eur Physiopathol Respir 1986; 22: 217 224.

12. Masip AV, Alonso Gómez AM, Martín Durán R, Moreno Yagüela M, Oliver Ruiz JM. Rodríguez Radial JM, et al. Guías de práctica clínica de la Sociedad Española de Cardiología en ecocardiografía. Rev Esp Cardiol 2000; 53: 663-83.

13. Damarla M, Celli B, Mullerova H, Pinto-Plata-V. Discrepancy in the use of confirmatory tests in patients hospitalized with the diagnosis of chronic obstructive pulmonary disease or congestive heart failure. Respir Care 2006; 51: 1120-1124.

14. Naberan K, De la Roza C, Lamban M, Gobartt E, Martin A, Miravitlles M. Utilización de la espirometría en el diagnóstico y tratamiento de la EPOC en atención primaria. Arch Bronconeumol 2006; 42: 638-644.

15. Bolton CE, Ionescu AA, Edwards PH, Faulkner TA, Edwards SM, Shale DJ. Attaining a correct diagnosis of COPD in general practice. Res Med 2005; 99: 493-500.

16. Han M, Kim MG, Mardon R, Renner Ph, Sullivan S, Diette G, et al. Spirometry utilization for COPD: How do we measure up? Chest; 2007 (publish online)

17. Miravitlles M, Murio M, Guerrero T, Gisbert R. Tratamiento de la bronquitis crónica y de la EPOC en atención primaria. Arch Bronconeumol 1999; 35: 173-178. 